\title{
Changing malaria intervention coverage, transmission and hospitalization in Kenya
}

Emelda A Okiro ${ }^{1,2^{*}}$, Victor A Alegana ${ }^{1}$, Abdisalan M Noor ${ }^{1,2}$, Robert W Snow ${ }^{1,2}$

\begin{abstract}
Background: Reports of declining incidence of malaria disease burden across several countries in Africa suggest that the epidemiology of malaria across the continent is in transition. Whether this transition is directly related to the scaling of intervention coverage remains a moot point.

Methods: Paediatric admission data from eight Kenyan hospitals and their catchments have been assembled across two three-year time periods: September 2003 to August 2006 (pre-scaled intervention) and September 2006 to August 2009 (post-scaled intervention). Interrupted time series (ITS) models were developed adjusting for variations in rainfall and hospital use by surrounding communities to show changes in malaria hospitalization over the two periods. The temporal changes in factors that might explain changes in disease incidence were examined sequentially for each hospital setting, compared between hospital settings and ranked according to plausible explanatory factors.

Results: In six out of eight sites there was a decline in Malaria admission rates with declines between 18\% and $69 \%$. At two sites malaria admissions rates increased by $55 \%$ and $35 \%$. Results from the ITS models indicate that before scaled intervention in September 2006, there was a significant month-to-month decline in the mean malaria admission rates at four hospitals (trend $P<0.05$ ). At the point of scaled intervention, the estimated mean admission rates for malaria was significantly less at four sites compared to the pre-scaled period baseline. Following scaled intervention there was a significant change in the month-to-month trend in the mean malaria admission rates in some but not all of the sites. Plausibility assessment of possible drivers of change pre- versus post-scaled intervention showed inconsistent patterns however, allowing for the increase in rainfall in the second period, there is a suggestion that starting transmission intensity and the scale of change in ITN coverage might explain some but not all of the variation in effect size. At most sites where declines between observation periods were documented admission rates were changing before free mass ITN distribution and prior to the implementation of ACT across Kenya.

Conclusion: This study provides evidence of significant within and between location heterogeneity in temporal trends of malaria disease burden. Plausible drivers for changing disease incidence suggest a complex combination of mechanisms, not easily measured retrospectively.
\end{abstract}

\section{Background}

Reports of declining incidence of malaria hospitalization, deaths and prevalence in several, diverse areas of Africa suggest that the epidemiology of malaria is in transition across the continent [1-17]. This transition has been coincidental with the scaling of intervention coverage

\footnotetext{
* Correspondence: eokiro@nairobi.kemri-wellcome.org

${ }^{1}$ Malaria Public Health \& Epidemiology Group, Centre for Geographic Medicine Research - Coast, Kenya Medical Research Institute/Wellcome Trust Research Programme, P.O. Box 43640, 00100 GPO, Nairobi, Kenya Full list of author information is available at the end of the article
}

and increased international funding for the control of malaria. However, precise attribution to intervention coverage alone remains circumstantial, not least because at most sites where declining disease incidence and prevalence have been reported, declines started before significant increase in donor assistance and scaled intervention coverage [2,8,9,12-14].

Adjusted trends in inpatient paediatric malaria case burden over 10 years in a sample of 17 hospitals with varied malaria transmission ecologies across Kenya were recently described using population-adjusted clinical 
data from defined hospital catchment areas [17]. The results showed divergent temporal patterns of disease incidence between sites. Importantly these data signalled that all was not equal across a single country. Here the possible mechanisms underlying the differences between these sites are explored in more detail in an effort to explain plausible drivers for changing disease incidence.

\section{Methods and Results}

Overview of sample selection, time-periods and methodological approach

For the purposes of evaluating mechanisms for change, hospital settings with adequate temporal data on insecticide-treated net (ITN) coverage (intervention change) and parasite prevalence (transmission intensity change) within defined spatial areas around hospitals that serve as the catchment to these hospitals were selected. Of the 17 original hospital settings the final selection of eight hospitals covered the dominant malaria ecologies that characterize Kenya including: three hospitals Bungoma, Kisumu, and Siaya District General Hospitals (DGH) in the Western/lakeside high transmission areas; Kericho and Kisii DGH located in the Highlands with typically epidemic transmission; and the three sites in Coastal Kenya: Malindi, Kilifi and Msambweni DGH.

Data have been assembled across two three-year time periods: September 2003 to August 2006 and September 2006 to August 2009: corresponding to important timelines for malaria intervention and drug policy change in Kenya. A range of plausible drivers and effect modifiers of changes in disease incidence were identified including intervention coverage, rainfall, service use and malaria transmission intensity. These data have been assembled at different spatial resolutions to match the communities served by the hospitals used to define disease incidence.

Defining attribution is fraught with many challenges and "plausibility designs" are often regarded as the only feasible option to evaluate the impact of nationally promoted large-scale intervention or changing risks $[18,19]$. These approaches have been recently promoted as a means of examining changes consequent upon scaled malaria control effort in sub-Saharan Africa [20]. Initially, temporal changes in factors that might explain changes in disease incidence were sequentially examined for each hospital setting, and were compared between hospital settings and plausible explanatory factors were ranked relative to proportional changes in disease incidence. An analytical procedure called intervention analysis was then used, this allows some exogenous event, in this case increasing ownership of ITNs and or a change in the first-line treatment policy to ACT, to occur that would affect the behaviour of the time-series of disease incidence being modelled as an ARMA process using the segmented regression [21,22].
Defining the geographic scope of attribute data - hospital catchment populations

High resolution census data were used to produce population distribution estimates around each hospital and combined with a random sample of admissions where residence was defined to compute distance travelled from their homes to the hospital. Thematic maps were created in ARCGIS 9.1 (ESRI, Inc., Redland, CA, USA) and used to define a minimum catchment of census bureau enumeration areas (EA) that would capture more than $90 \%$ of all admissions to the paediatric wards of each hospital [17]. Population counts in each EA where then projected from the 1999 census using growth rate curves and corrected for the proportion of the population aged less than 15 years [23]. The $0-14$ year projected population estimates were used to compute the person-years-at-risk (PYAR) for the mid years $2004 / 5$ and $2007 / 8$ to estimate PYAR in the 2003-2006 and 2006-2009 periods respectively.

\section{Paediatric admission data}

Inpatient hospital registers were arranged serially to represent a continuous, uninterrupted series. Each admission entry in the registers was recorded on a tally sheet indicating the month of admission, whether a primary working diagnosis of malaria was assigned to the child and whether the child was aged less than 15 years. Admissions without a diagnosis of malaria were coded similarly. In six hospitals, slide-confirmed malaria diagnoses at admission were not universally available within and between hospital sites for the surveillance period, however most admissions are likely to have a blood film prepared pre-admission, but the parasitological results could not be linked to patient records or registers and this continues to pose a significant information gap. Therefore our working definitions of "malaria" were patients admitted with a diagnosis of malaria, probably managed clinically as malaria during their admission but without documented parasitological confirmation. At Siaya and Kilifi DGHs a more detailed clinical admission criteria have been used since 2003 and described elsewhere [24-26]. Data were missing for two months (September \& October 2003) at Bungoma and admission numbers were modeled in STATA (version 10.1) by creating multiple imputed data sets for missing values using other existing variables (rainfall and the number of malaria and non-malaria deaths) for these missing data [17]. Malaria and non-malaria incidence was computed using the estimated children aged 0-14 years resident in the hospital catchment area and annualized over three years for the "baseline", pre-scaled intervention period September 2003-August 2006 and post-scaled intervention period September 2006-August 2009. Monthly malaria and non-malaria incidence rates were 
computed for each month of observation using the estimated annual proportion of the population aged less than 15 years.

An ARMAX model was then applied [21] with changes in service use (captured by non-malaria admission rates) included as an explanatory variable resulting in a predicted or smoothed malaria admission rate per month for each hospital site over the period 2003 to 2009 (Figure 1). The ARMAX model was then extended to include an interruption in the series to identify permanent changes in the level of the disease incidence series (interrupted time series analysis; ITS), that fits a linear regression to each segment, pre- and post- period of scaled intervention. The model is structured to identify changes in the level of the series $[21,22,27]$, with intervention times entered as explanatory variables in the model. We have used an exact intervention time point corresponding to the intersection between the two time periods described above. Segmented regression analyses were then used to estimate the relative change in malaria admissions after scaled intervention compared with before, taking into account pre-ITN malaria admissions and trend fitting different means for each period.

During the six years of surveillance there were a total of 166,632 paediatric admissions including 78,530 (47\%) admissions where a diagnosis included malaria. The proportion of admissions attributed to malaria across the entire surveillance period varied from $70 \%$ in Western/ lakeside, $45 \%$ in the Highlands and $22 \%$ along the Kenyan Coast (Table 1).

The annualized rate of paediatric malaria admission declined in the second observation period compared to the first at three locations on the coast (Table 1); the absolute decline (expressed as the incidence rate difference of malaria per 1000 between the periods 2003-2006 and 2006-2009) was greatest in Malindi (-5.07) and lowest in Msambweni $(-0.82)$. These are equivalent to decline of $69 \%$ in Kilifi, $61 \%$ in Malindi to the smallest drop at $18 \%$ in Msambweni (Table 1; incidence rate ratio (IRR)). Reductions in the second period compared to the first were also observed for the two sites in the highlands Kericho (-4.30) and Kisii (-10.85) and one urban site close to Lake Victoria, Kisumu (-9.08) (Table 1). Conversely, malaria admissions rates increased by $55 \%$ and $35 \%$ at Bungoma and Siaya respectively (IRR; Table 1). The incidence rate differences per 1000 children 0-14 between the periods 2003-2006 and 2006-2009 in nonmalaria admission rates were minimal at all sites except Malindi and Msambweni where the differences were 1.9 and 1.78, respectively (incidence rate difference; Table 1).

The relationship between malaria admissions by month shows declines in Kilifi, Malindi, Msambweni, Kisii, Kericho and only in the first period in Kisumu (Figure 1).
Importantly the time trends show that the declines started before scaled intervention in Kilifi, Malindi, Kisii, Kericho and Kisumu. This appears less clear in Msambweni. Conversely the trend in malaria admission rates showed a rise at Bungoma and Siaya with a rise in cases occurring largely in 2008 and 2009. At Kisumu, somewhat of an outlier, following the decline in the first period there was a rise in malaria admission rates in the second period occurring in 2009 (Figure 1). Results from the interrupted time series analysis indicate that just before the beginning of the observation period, malaria admission rates on average were between 2.21 to 12.5 per 1000 children $0-14$ per month (mean 6.30). Before September 2006, there was a significant month-to-month change in the mean number of malaria admission rates in Kisumu, Kilifi, Malindi and Msambweni with a significant downward trend (P-value for baseline trend $<0.05$ ) (Table 2). Immediately after the scaled-intervention, the estimated mean admission rates for malaria was significantly less by $0.52,0.59$, and 0.13 in Kisumu, Kisii and Msambweni but were not significant at the other sites. There was no significant change in the month-to-month trend in the mean admission rates for malaria across most of the hospitals except in Bungoma, Siaya, Kisumu and Msambweni where the month-tomonth trend in the mean malaria admission rates increased significantly in the second period ( $\mathrm{P}$-value for trend change $<0.05$ ) (Table 2).

\section{Rainfall}

To examine the effects of rainfall, an important determinant of malaria transmission, monthly precipitation data were obtained from meteorological stations located within the catchment areas of six hospitals. Because records were incomplete or unavailable, rainfall data was obtained from the nearest possible metrological station with complete data for Msambweni, Bungoma, Kilifi and Siaya $(32 \mathrm{~km}$ to $70 \mathrm{~km}$ from the catchment boundary respectively). Continuous mean monthly and annual rainfall and the number of months in a year with rainfall $>80 \mathrm{~mm}$ or $>60 \mathrm{~mm}$ among other variables were examined. There was an increasing trend toward higher annual precipitation in the second observation period (2006-2009) compared to the first observation period (2003-2006). Between September 2006 and August 2009, the mean monthly rainfall in seven out of the eight study sites was higher on average than the three years prior to this period (Table 3 and 4). The mean difference in average monthly rainfall between the first and second observation periods was $19.5 \mathrm{~mm}$ (median 19.2; IQR 11.2-25.9). In addition, the number of three-month periods of continuous rainfall above $60 \mathrm{~mm}$ indicates that the period before 2006 was notably drier than the period after 2006 (Additional file 1 Table S1). 

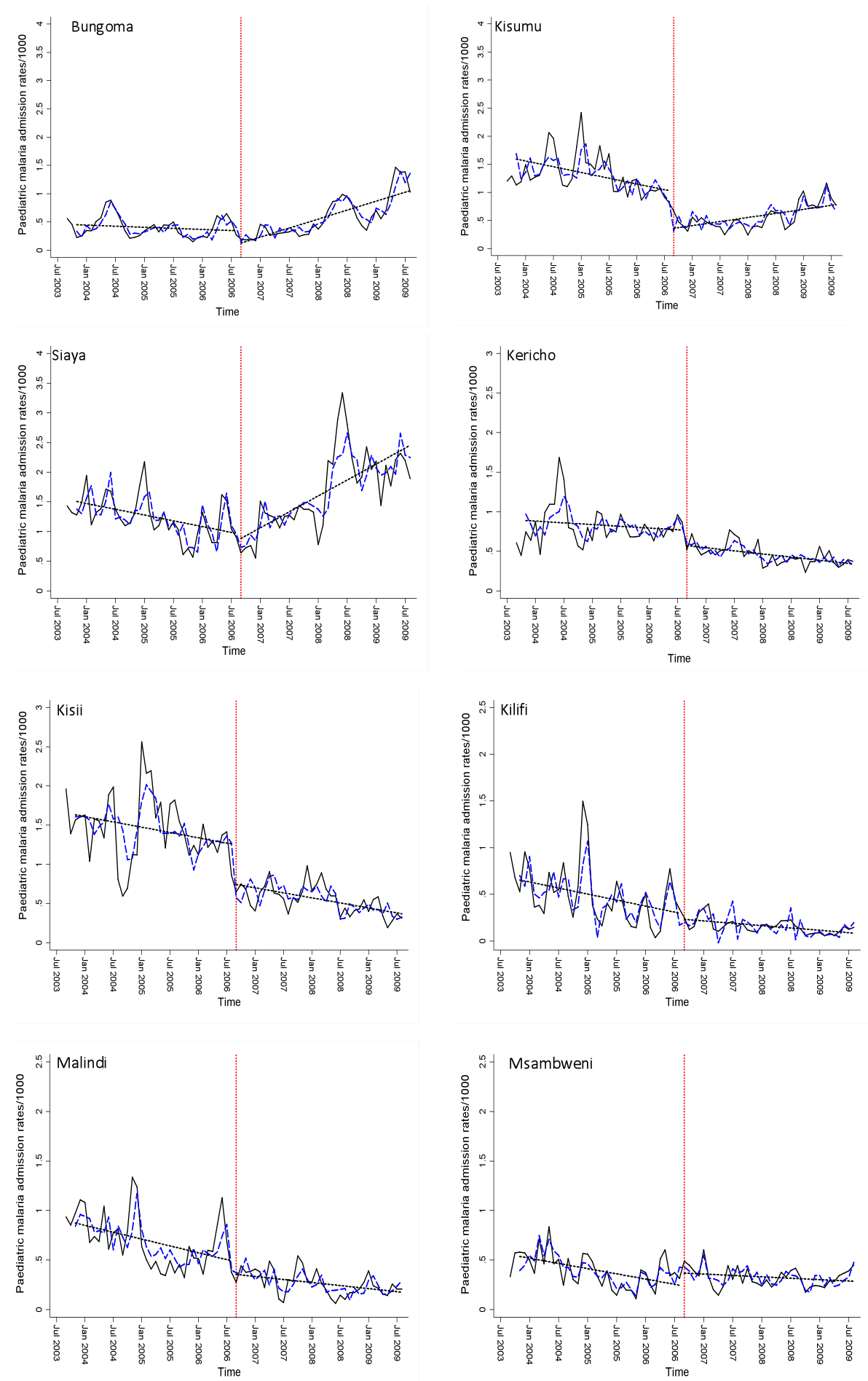

Figure 1 Graph panels show changing paediatric hospitalizations rates due to malaria per 1000 children $0-14$ years before and after scaling up of interventions. The data represents paediatric malaria admission rates by month (black solid line); model predictions of paediatric malaria hospitalization rates controlling for non-malaria case rates, rainfall and controlling for autoregressive and moving average effects (dashed blue line). Fitted lines illustrate the linear trends from model predictions (dashed line) for each segment of the period separated by a break point (red dashed vertical line). 
Table 1 Temporally aggregated paediatric admission data for malaria each of the 8 hospitals between 2003- 2006 and 2006-2009 expressed per 1000 children aged 0-14 years at risk per annum and 95\% confidence intervals computed using a Poisson distribution

\begin{tabular}{|c|c|c|c|c|c|c|}
\hline $\begin{array}{l}\text { Hospital } \\
\text { location }\end{array}$ & $\begin{array}{l}\text { Average malaria } \\
\text { admission rate/1000 } \\
\text { Sep } 2003 \text { - Aug } 2006 \\
\text { [95\% Cl] (number of } \\
\text { malaria admissions) }\end{array}$ & $\begin{array}{l}\text { Average malaria } \\
\text { admission rate/1000 } \\
\text { Sep } 2006 \text { - Aug } 2009 \\
\text { [95\% Cl] (number of } \\
\text { malaria admissions) }\end{array}$ & $\begin{array}{l}\text { Inc Rate } \\
\text { Ratio (95\% } \\
\text { Cl) [Inc } \\
\text { Rate Diff ] } \\
(95 \% \mathrm{Cl})\end{array}$ & $\begin{array}{l}\text { Average non-malaria } \\
\text { admission rate/1000 Sep } \\
2003 \text { - Aug } 2006 \text { [95\% } \\
\text { CI] (number of non- } \\
\text { malaria admissions) }\end{array}$ & $\begin{array}{l}\text { Average nonmalaria } \\
\text { admission rate/1000 Sep } \\
2006 \text { - Aug } 2009[95 \% \\
\mathrm{Cl}] \text { (number of non- } \\
\text { malaria admissions) }\end{array}$ & $\begin{array}{l}\text { Inc Rate } \\
\text { Ratio (95\% } \\
\text { Cl) [Inc } \\
\text { Rate Diff ] } \\
(95 \% \mathrm{Cl}) \\
\end{array}$ \\
\hline \multicolumn{7}{|c|}{ Western/Lakeside } \\
\hline $\begin{array}{l}\text { Bungoma } \\
\text { DGH }\end{array}$ & $\begin{array}{l}4.66 \\
{[4.53-4.79]} \\
(4,853)\end{array}$ & $\begin{array}{l}7.21 \\
{[7.06-7.37]} \\
(8,500)\end{array}$ & $\begin{array}{l}1.55 \\
(1.50-1.61) \\
{[2.56](2.36-} \\
2.76)\end{array}$ & $\begin{array}{l}3.63 \\
{[3.52-3.75]} \\
(3,783)\end{array}$ & $\begin{array}{l}3.45 \\
{[3.34-3.56]} \\
(4,063)\end{array}$ & $\begin{array}{l}0.95 \\
(0.91-0.99) \\
{[-0.19]} \\
(-0.34- \\
-0.03)\end{array}$ \\
\hline $\begin{array}{l}\text { Kisumu } \\
\text { DGH }\end{array}$ & $\begin{array}{l}15.89 \\
{[15.61-16.18]} \\
(12,128)\end{array}$ & $\begin{array}{l}6.81 \\
{[6.64-6.99]} \\
(5,578)\end{array}$ & $\begin{array}{l}0.43 \\
(0.42-0.44) \\
{[-9.08]} \\
(-9.41- \\
-8.74)\end{array}$ & $\begin{array}{l}3.28 \\
{[3.15-3.41]} \\
(2,501)\end{array}$ & $\begin{array}{l}3.28 \\
{[3.16-3.41]} \\
(2,685)\end{array}$ & $\begin{array}{l}1.00 \\
(0.95-1.06) \\
{[0.00]} \\
(-0.18-0.18)\end{array}$ \\
\hline $\begin{array}{l}\text { Siaya } \\
\text { DGH }\end{array}$ & $\begin{array}{l}14.93 \\
{[14.52-15.35]} \\
(5,042)\end{array}$ & $\begin{array}{l}20.12 \\
{[19.65-20.60]} \\
(6,982)\end{array}$ & $\begin{array}{l}1.35 \\
(1.30-1.40) \\
{[5.19]} \\
(4.56-5.82)\end{array}$ & $\begin{array}{l}8.10 \\
{[7.80-8.41]} \\
(2,736)\end{array}$ & $\begin{array}{l}7.22 \\
{[6.94-7.51]} \\
(2,506)\end{array}$ & $\begin{array}{l}0.89 \\
(0.84-0.94) \\
{[-0.88]} \\
(-1.30- \\
-0.47) \\
\end{array}$ \\
\hline \multicolumn{7}{|l|}{ Highlands } \\
\hline $\begin{array}{l}\text { Kericho } \\
\text { DGH }\end{array}$ & $\begin{array}{l}9.80 \\
{[9.55-10.05]} \\
5,923\end{array}$ & $\begin{array}{l}5.50 \\
{[5.32-5.68]} \\
(3,580)\end{array}$ & $\begin{array}{l}0.56 \\
(0.54-0.59) \\
{[-4.30]} \\
(-4.30- \\
-3.99)\end{array}$ & $\begin{array}{l}7.21 \\
7.00-7.43 \\
4,360\end{array}$ & $\begin{array}{l}6.58 \\
{[6.38-6.78]} \\
(4,281)\end{array}$ & $\begin{array}{l}0.91 \\
(0.87-0.95) \\
{[-0.64]} \\
(-0.93- \\
-0.35)\end{array}$ \\
\hline $\begin{array}{l}\text { Kisii } \\
\text { DGH }\end{array}$ & $\begin{array}{l}17.45 \\
{[17.12-17.79]} \\
(10,450)\end{array}$ & $\begin{array}{l}6.60 \\
{[6.40-6.80]} \\
(4,201)\end{array}$ & $\begin{array}{l}0.38 \\
(0.36-0.39) \\
{[-10.85]} \\
(-11.24- \\
-10.46) \\
\end{array}$ & $\begin{array}{l}16.67 \\
{[16.35-17.00]} \\
(9,983)\end{array}$ & $\begin{array}{l}17.28 \\
{[16.95-17.60]} \\
(10,995)\end{array}$ & $\begin{array}{l}1.04 \\
(1.01-1.06) \\
{[0.60]} \\
(0.15-1.06)\end{array}$ \\
\hline \multicolumn{7}{|l|}{ Coastal } \\
\hline $\begin{array}{l}\text { Kilifi } \\
\text { DGH }\end{array}$ & $\begin{array}{l}5.98 \\
{[5.78-6.18]} \\
(3,345)\end{array}$ & $\begin{array}{l}1.85 \\
{[1.75-1.97]} \\
(1,139)\end{array}$ & $\begin{array}{l}0.31 \\
(0.29-0.33) \\
{[-4.12]} \\
(-4.35- \\
-3.89) \\
\end{array}$ & $\begin{array}{l}19.61 \\
{[19.24-19.98]} \\
10,974\end{array}$ & $\begin{array}{l}19.10 \\
{[18.75-19.45]} \\
(11,730)\end{array}$ & $\begin{array}{l}0.97 \\
(0.95-1.00) \\
{[-0.51]} \\
(-1.01- \\
-0.00) \\
\end{array}$ \\
\hline $\begin{array}{l}\text { Malindi } \\
\text { DGH }\end{array}$ & $\begin{array}{l}8.28 \\
{[7.96-8.61]} \\
(2,573)\end{array}$ & $\begin{array}{l}3.21 \\
{[3.03-3.41]} \\
(1,105)\end{array}$ & $\begin{array}{l}0.39 \\
(0.36-0.42) \\
{[-5.07]} \\
(-5.44- \\
-4.70) \\
\end{array}$ & $\begin{array}{l}18.76 \\
{[18.28-19.25]} \\
5,828\end{array}$ & $\begin{array}{l}16.88 \\
{[16.45-17.32]} \\
(5,807)\end{array}$ & $\begin{array}{l}0.90 \\
(0.87-0.93) \\
{[-1.88]} \\
(-2.53- \\
-1.23) \\
\end{array}$ \\
\hline $\begin{array}{l}\text { Msambweni } \\
\text { DGH }\end{array}$ & $\begin{array}{l}4.74 \\
{[4.51-4.97]} \\
(1,655)\end{array}$ & $\begin{array}{l}3.91 \\
{[3.71-4.12]} \\
(1,476)\end{array}$ & $\begin{array}{l}0.82 \\
(0.77-0.89) \\
{[-0.83]} \\
(-1.13- \\
-0.53)\end{array}$ & $\begin{array}{l}7.15 \\
{[6.88-7.44]} \\
2,498\end{array}$ & $\begin{array}{l}8.93 \\
{[8.63-9.24]} \\
(3,372)\end{array}$ & $\begin{array}{l}1.25 \\
(1.19-1.32) \\
{[1.78]} \\
(1.37-2.19)\end{array}$ \\
\hline
\end{tabular}

\section{Parasite prevalence}

One of the most widely used measures of Plasmodium falciparum malaria transmission intensity is the parasite rate (PfPR) measured through community-based surveys and expressed as the proportion of people infected at one point in time. A comprehensive dataset has been assembled of all geo-located PfPR survey data undertaken in Kenya since 1974 [28]. These data have been re-sampled to identify surveys undertaken within each hospital catchment, between 2002 and 2010 where more than 50 people had been surveyed. The age-ranges reported varied between surveys and these were standardized to a single age range 2-10 years $\left(P f \mathrm{PR}_{2-10}\right)$ using algorithms described elsewhere [29]. The 
Table 2 Parameter estimates, confidence intervals and P-values from the full segmented ARMAX regression models predicting mean monthly numbers of malaria cases per month in eight hospitals over time

\begin{tabular}{|c|c|c|c|c|c|}
\hline & & Mean before scale up & Baseline trend & Mean after scale up & Trend change after scale up \\
\hline \multicolumn{6}{|l|}{ Western/Lakeside } \\
\hline & Coefficient & 2.2189 & -0.0038 & -0.0797 & 0.0251 \\
\hline \multirow[t]{4}{*}{ Bungoma DGH } & $95 \% \mathrm{Cl}$ & $-5.8433-$ & $-0.0185-$ & $-0.4578-$ & $0.0043-$ \\
\hline & $P$-value & 0.2811 & 0.0109 & 0.2985 & 0.0459 \\
\hline & & 0.5900 & 0.6100 & 0.680 & 0.018 \\
\hline & & 12.5782 & -0.0213 & -0.5247 & 0.0292 \\
\hline \multirow[t]{4}{*}{ Kisumu DGH } & Coefficient & $5.7537-$ & $-0.0341--$ & $-0.8117--$ & $0.0062-$ \\
\hline & $95 \% \mathrm{Cl}$ & 19.4028 & 0.0086 & 0.2377 & 0.0521 \\
\hline & $P$-value & 0.000 & 0.001 & 0.000 & 0.013 \\
\hline & & 7.3604 & -0.0125 & -0.1275 & 0.0557 \\
\hline \multirow[t]{3}{*}{ Siaya DGH } & Coefficient & $-7.9641-$ & $-0.0405-$ & $-0.9069-$ & $0.0138-$ \\
\hline & $95 \% \mathrm{Cl}$ & 22.6849 & 0.0156 & 0.6519 & 0.0975 \\
\hline & $P$-value & 0.347 & 0.384 & 0.749 & 0.009 \\
\hline \multicolumn{6}{|l|}{ Highlands } \\
\hline & & 2.9036 & -0.0043 & -0.1847 & -0.0014 \\
\hline \multirow[t]{4}{*}{ Kericho DGH } & Coefficient & $-3.1163-$ & $-0.0156-$ & $-0.5648-$ & $-0.0210-$ \\
\hline & $95 \% \mathrm{Cl}$ & 8.9234 & 0.0070 & 0.1954 & 0.0182 \\
\hline & $P$-value & 0.344 & 0.457 & 0.341 & 0.888 \\
\hline & & 6.10113 & -0.00892 & -0.59444 & 0.00019 \\
\hline \multirow[t]{3}{*}{ Kisii DGH } & Coefficient & $-0.3973-$ & $-0.0209-$ & $-1.0789--$ & $-0.0148-$ \\
\hline & $95 \% \mathrm{Cl}$ & 12.5996 & 0.0031 & 0.1100 & 0.0152 \\
\hline & $P$-value & 0.066 & 0.146 & 0.016 & 0.980 \\
\hline \multicolumn{6}{|l|}{ Coastal } \\
\hline & & 7.2789 & -0.0131 & -0.0382 & 0.0094 \\
\hline \multirow[t]{4}{*}{ Kilifi DGH } & Coefficient & $3.3066-$ & $-0.0207--$ & $-0.3036-$ & $-0.0069-$ \\
\hline & $95 \% \mathrm{Cl}$ & 11.2512 & 0.0056 & 0.2272 & 0.0256 \\
\hline & $P$-value & 0.000 & 0.001 & 0.778 & 0.260 \\
\hline & & 7.6455 & -0.0134 & -0.0587 & 0.0072 \\
\hline \multirow[t]{4}{*}{ Malindi DGH } & Coefficient & $2.6559-$ & $-0.0229--$ & $-0.3656-$ & $-0.0111-$ \\
\hline & $95 \% \mathrm{Cl}$ & 12.6351 & 0.0040 & 0.2483 & 0.0255 \\
\hline & $P$-value & 0.003 & 0.005 & 0.708 & 0.441 \\
\hline & Coefficient & 4.3503 & -0.0078 & -0.1287 & 0.0143 \\
\hline \multirow[t]{3}{*}{ Msambweni DGH } & $95 \% \mathrm{Cl}$ & $2.7546-$ & $-0.0108--$ & $-0.2452--$ & $0.0080-$ \\
\hline & $P$-value & 5.9459 & 0.0048 & 0.0122 & 0.0206 \\
\hline & & 0.000 & 0.000 & 0.030 & 0.000 \\
\hline
\end{tabular}

prevalence estimates across the two time periods were summarized using the median and inter-quartile ranges of age standardized prevalence estimates ( $P f \mathrm{PR}_{2-10}$ ) across the 300 surveys identified as having been undertaken within the catchment areas since 2002 (Additional file 1 Table S2).

During the first observation period, before scaled intervention coverage in September 2006, the median $P f \mathrm{PR}_{2-10}$ was highest around Lake Victoria. At Kisumu median $P f \mathrm{PR}_{2-10}$ was $71 \%$ and around Siaya $\mathrm{DGH} P f \mathrm{PR}_{2-}$ 10 was $55 \%$ (Table 3 ), however it was reportedly lower in Bungoma during this period (median $P f \mathrm{PR}_{2-10}, 15 \%$;
Table 3). In the highlands median $P f \mathrm{PR}_{2-10}$ was $22 \%$ at Kisii and 5\% at Kericho (Table 3). Along the Kenyan coast median PfPR2-10 was between 10 and 13\% during the first, pre-scaled intervention period (Table 3 ). By the second period (2006-2009) median $P f \mathrm{PR}_{2-10}$ had declined in six out of the eight sites, this decline varied between sites (Table 4) and the median Pf $\mathrm{PR}_{2-10}$ had increased to over $45 \%$ at Bungoma and rose slightly at Msambweni from $10 \%$ to $12 \%$ (Table 4). During the second observation period two hospital catchment areas had a median $P f \mathrm{PR}_{2-10}$ of greater than $40 \%$ : Bungoma and Siaya (Table 4). 
Table 3 Characteristics of Hospital Catchment areas 2003-2006

\begin{tabular}{|c|c|c|c|c|c|c|c|c|}
\hline HOSPITAL & $\begin{array}{l}\text { Projected } \\
\text { Population } \\
<15 \text { years } \\
\text { in } 2003\end{array}$ & $\begin{array}{l}\text { Average annual } \\
\text { number of Total } \\
\text { admissions } \\
(2003-2006)\end{array}$ & $\begin{array}{l}\text { Average } \\
\text { annual } \\
\text { Rainfall }(\mathrm{mm}) \\
\text { at Baseline } \\
(2003-2006)\end{array}$ & $\begin{array}{l}\text { Median Age- } \\
\text { Corrected Parasite } \\
\text { Prevalence Jan } \\
\text { 2002-Aug } 2006^{1}\end{array}$ & $\begin{array}{l}\text { ITN } \\
\text { Coverage }\end{array}$ & $\begin{array}{l}\text { SP Success } \\
\text { Rate } \\
\text { Average \% } \\
\text { ACPR d14 } \\
(2000- \\
2003)^{2}\end{array}$ & $\begin{array}{l}\text { Number } \\
\text { reporting } \\
\text { fever in } \\
\text { last } 14 \\
\text { days DHS } \\
2003^{3}\end{array}$ & $\begin{array}{l}\text { Number (\%) of } \\
\text { fevers seeking any } \\
\text { treatment from a } \\
\text { Public Health } \\
\text { Facility DHS } 2003^{3}\end{array}$ \\
\hline
\end{tabular}

\begin{tabular}{|c|c|c|c|c|c|c|c|c|}
\hline $\begin{array}{l}\text { Western/ } \\
\text { Lakeside }\end{array}$ & & & & & & & & \\
\hline $\begin{array}{l}\text { Bungoma } \\
\text { DGH }\end{array}$ & 326137 & 2879 & 1337.0 & $15.3 \%$ & $20.5 \%$ & & & \\
\hline Kisumu DGH & 245600 & 4876 & 1241.2 & $70.6 \%$ & $26.2 \%$ & $55.4-83.1$ & $733^{4}$ & $168(22.9)^{4}$ \\
\hline Siaya DGH & 111026 & 2593 & 1241.2 & $54.7 \%$ & $21.0 \%$ & & & \\
\hline Highlands & & & & & & & & \\
\hline Kericho DGH & 194090 & 3428 & 2130.5 & $5.2 \%$ & $14.3 \%$ & 70.37 & $718^{5}$ & $190(26.5)^{5}$ \\
\hline Kisii DGH & 193608 & 6811 & 1978.0 & $22.2 \%$ & $35.6 \%$ & & & \\
\hline Coastal & & & & & & & & \\
\hline Kilifi DGH & 178045 & 4773 & 1136.0 & $12.9 \%$ & $20.5 \%$ & & & \\
\hline Malindi DGH & 98401 & 2800 & 954.7 & $11.8 \%$ & $8.8 \%$ & $67.2-96.8$ & 262 & $100(38.2)$ \\
\hline $\begin{array}{l}\text { Msambweni } \\
\text { DGH }\end{array}$ & 111917 & 1384 & 588.7 & $10.4 \%$ & $15.1 \%$ & & & \\
\hline
\end{tabular}

Notes:

SP - Sulphadoxine-pyrimethamine ACPR - Acceptable Clinical \& Parasitological Responses by day 14.

${ }^{1}$ Includes data from surveys done in 2002 and school surveys done in January and February 2010 in Bungoma, Kisumu, Siaya and Kericho areas.

${ }^{2}$ Study references: [46]; [36,47].

${ }^{3}$ Estimates reported at the Provincial level.

${ }^{4}$ Combined estimates from Western and Nyanza provinces.

${ }^{5}$ Combined estimates from Rift Valley and Nyanza provinces.

\section{ITN coverage}

In 2001, the Kenyan Government adopted a policy on ITN to ensure $60 \%$ coverage by 2010 [30]. The dominant ITN delivery approach between 2003 and September 2006 was a combined full cost recovery retail sector promotion and subsidized cost ITN distribution using social marketing at rural clinics [31]. In 2006 the Ministry of Health, using funds from the Global Fund, launched a large-scale distribution campaign of free ITN to children under the age of five years, which was completed in September 2006 and rapidly increased equitable coverage of ITN nationwide [31]. Various household survey data have been collected in Kenya since 2003 aimed at defining the proportion of children below the age of five sleeping under an ITN during the night before the survey. Cluster level data congruent with the catchment areas defined for each of the eight hospitals were re-sampled from the national Demographic and Health Survey (DHS) undertaken in 2003 [32], Population Services International household sample surveys undertaken in 2005 and 2007 [33] and a national malaria indicator survey [34] undertaken in 2007. Each cluster was allocated to a time-period and data summed across clusters within each catchment. During the 2003 DHS survey only data on children was available but subsequent surveys included information on all age-groups and have therefore focussed on all-age use of ITN for comparisons between first and second observation periods.

During the first observation period bed net use by children aged less than five was universally low, the highest average coverage in 2003 was recorded in Kisumu (0.29\%). The average level of coverage across all eight sites was $20.3 \%$ in 2005 with the lowest level of coverage recorded in Malindi at 9\%, in Kericho and Msambweni coverage was $14 \%$ and $15 \%$ respectively while the highest estimated level of coverage was in Kisii at $37 \%$. By 2007, one year after the free mass distribution campaigns ITN coverage had increased considerably to an estimated average coverage of $41 \%$ across all sites ranging from lowest coverage in Kericho at 32\% to high of $50 \%$ in Malindi and $51 \%$ in Kisii. The average percentage increase was $21 \%$, however at Bungoma, Siaya, Kilifi and Kericho the increase in coverage was less than $20 \%$ and remained below $40 \%$ in all 4 sites including Msambweni during the post-scaled coverage period (Additional file 1 Table S3).

\section{Drug policy change and access to efficacious antimalarials}

Between 2000 and April 2006, sulphadoxine-pyrimethamine (SP) was the only drug available in most 
Table 4 Characteristics of Hospital Catchment areas 2006-2009

\begin{tabular}{|c|c|c|c|c|c|c|c|c|}
\hline HOSPITAL & $\begin{array}{l}\text { Projected } \\
\text { Population } \\
<15 \text { years } \\
\text { in } 2003\end{array}$ & $\begin{array}{l}\text { Average annual } \\
\text { number of Total } \\
\text { admissions (2003 - } \\
2006)\end{array}$ & 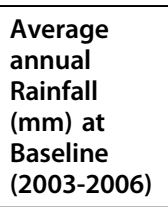 & $\begin{array}{l}\text { Median Age- } \\
\text { Corrected Parasite } \\
\text { Prevalence Jan } \\
\text { 2002-Aug } 2006^{1}\end{array}$ & $\begin{array}{l}\text { ITN } \\
\text { Coverage }\end{array}$ & $\begin{array}{l}\text { SP Success } \\
\text { Rate } \\
\text { Average \% } \\
\text { ACPR d14 } \\
(2000- \\
2003)^{2}\end{array}$ & $\begin{array}{l}\text { Number } \\
\text { reporting } \\
\text { fever in } \\
\text { last } 14 \\
\text { days DHS } \\
2003^{3}\end{array}$ & $\begin{array}{l}\text { Number (\%) of } \\
\text { fevers seeking any } \\
\text { treatment from a } \\
\text { Public Health } \\
\text { Facility DHS } 2003^{3}\end{array}$ \\
\hline \multicolumn{9}{|l|}{$\begin{array}{l}\text { Western/ } \\
\text { Lakeside }\end{array}$} \\
\hline $\begin{array}{l}\text { Bungoma } \\
\text { DGH }\end{array}$ & 417832 & 4188 & 1673.3 & $44.9 \%$ & $36.0 \%$ & & & \\
\hline Kisumu DGH & 282645 & 2754 & 1472.1 & $29.8 \%$ & $47.3 \%$ & 93.4- 100.0 & $686^{4}$ & $210(30.6)^{4}$ \\
\hline Siaya DGH & 111026 & 2593 & 1241.2 & $54.7 \%$ & $21.0 \%$ & & & \\
\hline \multicolumn{9}{|l|}{ Highlands } \\
\hline Kericho DGH & 225159 & 2620 & 2095.4 & $0.01 \%$ & $32.0 \%$ & N/A & $205^{5}$ & $205(35.7)^{5}$ \\
\hline Kisii DGH & 218698 & 5065 & 2063.0 & $1.9 \%$ & $51.1 \%$ & & & \\
\hline \multicolumn{9}{|l|}{ Coastal } \\
\hline Kilifi DGH & 214441 & 4290 & 1388.6 & $3.7 \%$ & $38.1 \%$ & & & \\
\hline Malindi DGH & 120658 & 2304 & 1145.6 & $4.5 \%$ & $49.9 \%$ & $87.0-100.0$ & 173 & $64(37.0)$ \\
\hline $\begin{array}{l}\text { Msambweni } \\
\text { DGH }\end{array}$ & 111917 & 1384 & 588.7 & $10.4 \%$ & $15.1 \%$ & & & \\
\hline \multicolumn{9}{|l|}{ Notes: } \\
\hline \multicolumn{9}{|c|}{ AL - Artmether-lumefathrine. } \\
\hline \\
\hline \multicolumn{2}{|c|}{ 'Includes data from school surveys done in January and February 2010 in Bungoma, Kisumu, Siaya and Kericho areas. } & \multicolumn{7}{|c|}{${ }^{2}$ Study references: [48-53]. } \\
\hline \multicolumn{9}{|c|}{$\begin{array}{l}{ }^{3} \text { Estimates reported at the Provincial level. These data were available at the provincial level and not specific to each district or catchment area and are presented as } \\
\text { such. }\end{array}$} \\
\hline \multicolumn{9}{|c|}{${ }^{4}$ Combined estimates from Western and Nyanza provinces. } \\
\hline
\end{tabular}

government clinics [35] however, the efficacy of SP declined rapidly over this period including empirical evidence at some of the selected hospital sites that demonstrated success rates of less than $75 \%$ by day 14 following treatment (Table 3, [36]; [37]). Officially first line treatment policy was changed in April 2004 to the more efficacious artemether-lumefathrine (AL) but the new policy was not effectively implemented until September 2006 [38]. The clinical and parasitological efficacy of AL was in excess of $94 \%$ in 2002-2003 at the coast [39] and several clinical trials undertaken since 2006 have shown AL efficacy to be in excess of $87 \%$. Information on SP and AL sensitivity was attributed to regions that broadly cover the catchments included in the analysis but it was not possible to re-assemble these data specifically to the communities served by the hospitals (Table 3 and 4).

Prior to 2006 SP was ubiquitously available in government facilities and through private retail providers [35,38]. However, AL implementation in 2006 limited access to only government and mission facilities [40] and the supply to these facilities has been erratic and incomplete [41]. Access to anti-malarials within 48 hours of onset of symptoms was generally poor across Kenya throughout the period of observation 2003-2009.
At four sentinel districts (including Msambweni and Kisii), Amin and colleagues showed that use of any antimalarial within 48 hours by febrile children was $15 \%$ in 2001 and $17 \%$ in 2006 [42]; unpublished data) and in the same sites this rose to only $23 \%$ by 2007 [43].

Household survey data on drug use is much less prolific than ITN usage data and, therefore, it was only possible to assemble national survey data at wider spatial resolutions than the catchment areas in the eight hospital sites. The respective provincial level data on treatments with an anti-malarial for children who had a reported fever in the last 14 days were assembled from the DHS 2003 survey and the MIS in 2007 to represent drug access in the two observation periods (Table 3 and 4). Between 2003 and 2006 the proportion of children with fever in the last two weeks who sought treatment from a Public Health Facility was estimated to be 23\% in Western/Lakeside region, 27\% in the Highlands and was highest at $38 \%$ on the Coast (Table 3). By the second period, four years after the survey, access to treatment had increased slightly across two study regions with the proportion of fevers seeking treatment from a Public Health Facility reported to be $31 \%$ in the Western/Lakeside and $36 \%$ in the Highlands. However, on 
Coast access to treatment has remained largely unchanged at 37\% in 2007 (Table 4).

\section{Comparison of health indictors pre- and post 2006}

The surveillance data were analysed by time to determine whether patterns in hospitalized malaria case incidence could be associated to equivalent changes in several important covariates. Outcome indicators are summarized as incidence rate ratios and incidence rate differences based on admission events with mid-period under 15 population estimates as the denominator. The relative change and the absolute changes $(95 \% \mathrm{CI})$ in covariates between period 1 and 2 calculated for each hospital were used as the comparative measures. The absolute change is used to describe the actual increase or decrease from the estimate in period one and is used to explore the magnitude of change. Relative change is used to describe changes between period one and two by comparing the absolute change to the reference value which here is the value of the estimate in the first period and has been used here to explore processes of causation specifically to examine the effect of changes in intervention coverage or climate variables on the occurrence of malaria cases. We examine changes in relation to the starting endemicity and baseline ITN coverage for the parasite prevalence and ITN coverage respectively.

Plausibility patterns exploring factors that might explain changes in disease incidence across hospital settings represented by different colors are shown in Figures 2 and 3. Patterns in IRR ( $\mathrm{x}$-axis) were examined against parasite prevalence in the first and second period, the absolute change in prevalence, ITN coverage in the second period and the absolute change in ITN coverage ( $\mathrm{x}$-axis). No obvious patterns were clearly evident however, considering that rainfall increased during the same period, out of all the factors we have examined, there is a suggestion that sustained high transmission intensity in the second period (Figure 2; middle graph) and the magnitude of change in ITN coverage (Figure 3; right graph) might explain some of the variation in effect size.

In addition, the combined effect of various factors were examined using the ranking of plausible explanatory factors (ranked by magnitude) with 1 representing the highest score/change and 8 the lowest score/change relative to the ranking of the effect size represented as the absolute change in disease incidence in each hospital site (Table 5). Despite the fact that the ranking shows largely ambiguous patterns; there is some indication that transmission intensity reduced to low levels in the sites that witnessed a substantial drop in malaria hospitalization (ranking 1-5) with the exception of Kisumu and Msambweni (Table 3 and 4). The ranking tables also showed that the two sites with a rise in malaria
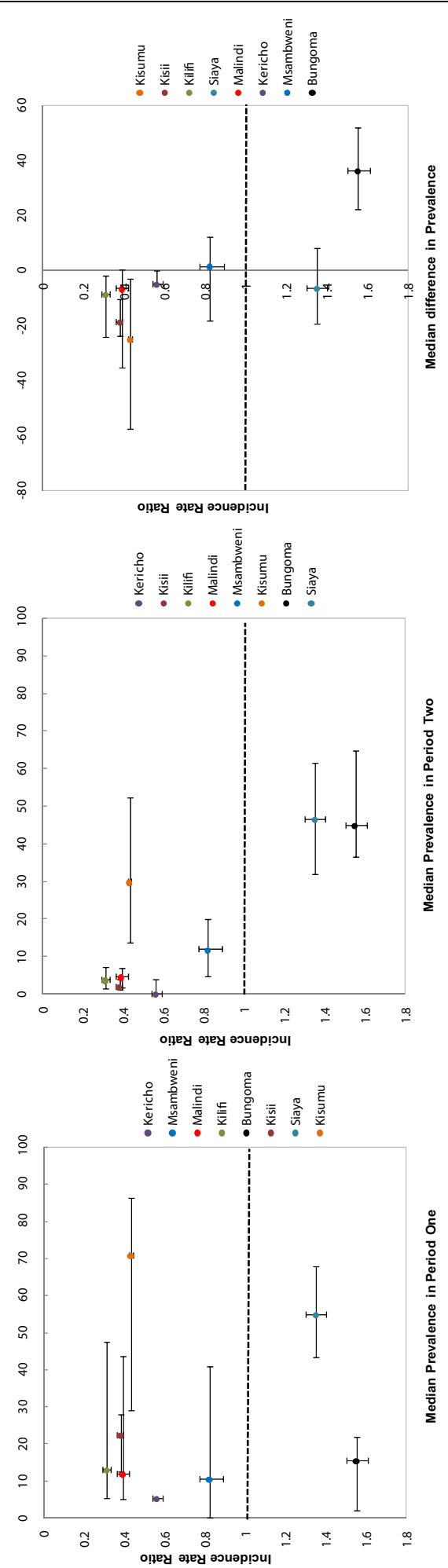

Figure 2 Relationship between changes in the incidence rate of malaria admissions between period one and two (Incidence rate ratio) and equivalent absoulte changes in transmission intensity across $\mathbf{8}$ hospital sites in Kenya (Left-starting PfPR; Middle - PfPR at the end of the study and Right - absolute difference in prevalence between period one and two). 

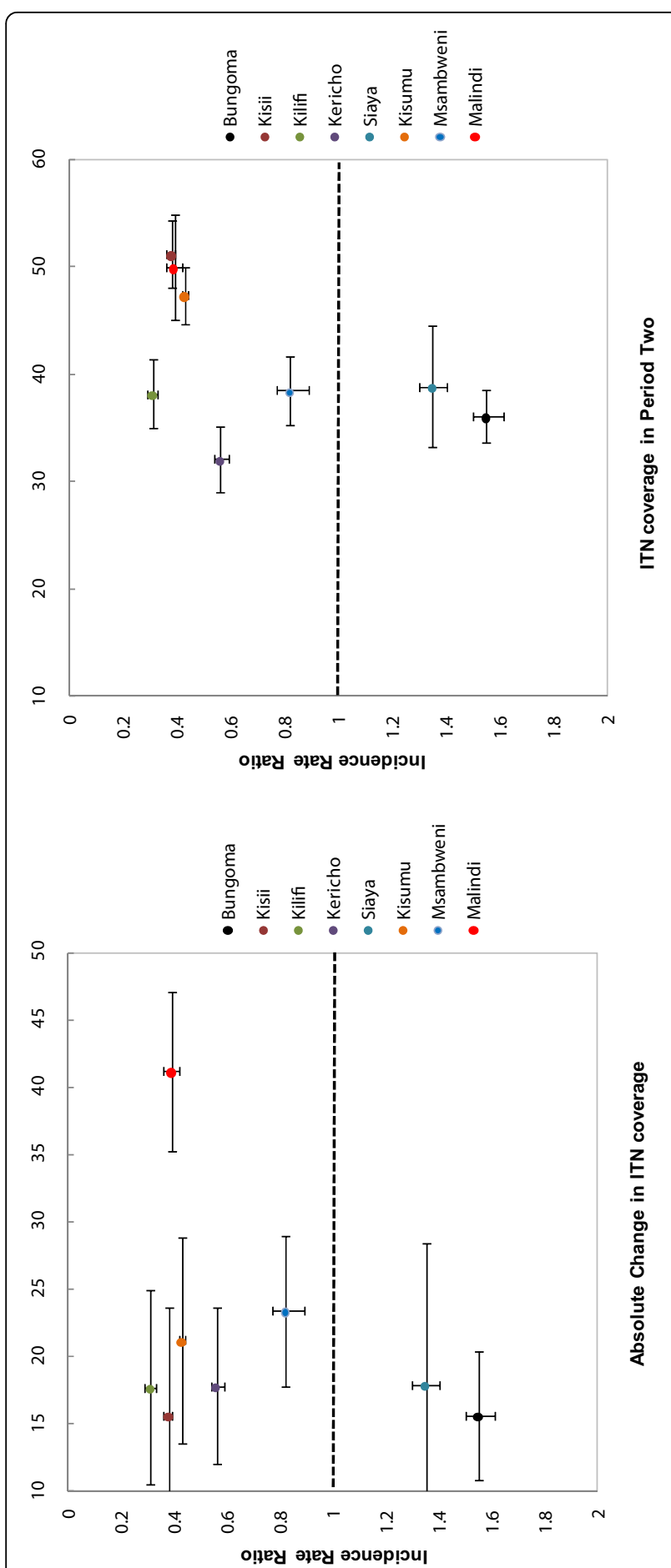

Figure 3 Relationship between changes in the incidence rate of malaria admissions between period one and two (Incidence rate ratio) and equivalent absolute changes in ITN coverage across 8 hospital catchments (Left- absolute difference in ITN coverage between period one and two; Right - ITN coverage in the follow-up period) hospitalization appear to have the highest transmission intensity in the second period (ranked 1 and 2); (Table 5). Kisii which had the highest absolute drop in malaria admission rates also had the highest level of ITN coverage in period two and a significant drop in $P f \mathrm{PR}_{2-10}$ (ranked 2).

\section{Discussion}

The temporal patterns of paeditaric malaria admissions at eight hospitals across the diverse malaria transmission ecology of Kenya were examined between two 36 month periods September 2003 and August 2009 and the period September 2006 to August 2009 representing two periods best described as reflecting major shifts in intervention policy change and scaled intervention. At six hospital sites (Kisumu, Kericho, Kisii, Kilifi, Malindi and Msambweni) hospitalization from malaria showed a significant reduction between these two time periods (Table 1), but in four cases the decline started before scaled intervention (significant baseline downward trend in Kilifi, Malindi Msambweni, and Kisumu, Table 2). Similar declines were observed at Msambweni on the southernmost tip of the Kenyan coast but these declines were less marked. Kisumu represents an outlier where despite the fact that there was a significant drop in malaria cases between period one and two hospitalization from malaria showed a significant upward trend in period two. At Siaya and Bungoma paediatric malaria admission rates rose in the second period compared to the pre-scaled intervention period before September 2006. It is important to acknowledge that the majority of the data analyzed here are not based upon parasitological confirmed cases of malaria as these data were often not available and of variable quality and accuracy and may have contributed to some systematic bias in the data and remains an inherent methodological limitation.

A number of extrinsic (rainfall), biological (transmission intensity) and intervention (ITN coverage and ACT access) that may have explained the changes in the interupted time-series of paediatric malaria admissions were examined. Rainfall proved not to explain any of the changes directly as the second observation period was wetter than the first at all sites where declines occurred and changes in rainfall patterns were not different between sites with increasing malaria admissions compared to those without (Table 3 and 4). Parasite prevalence during the period prior to scaled intervention among communities located within the catchments of the hospitals signalled that there was some tendency for those areas that showed the largest declines in admission rates in post-intervention to be of lowest 
Table 5 Plausibility ranking table indicating the ranking of each hospital by absolute change in several important factors

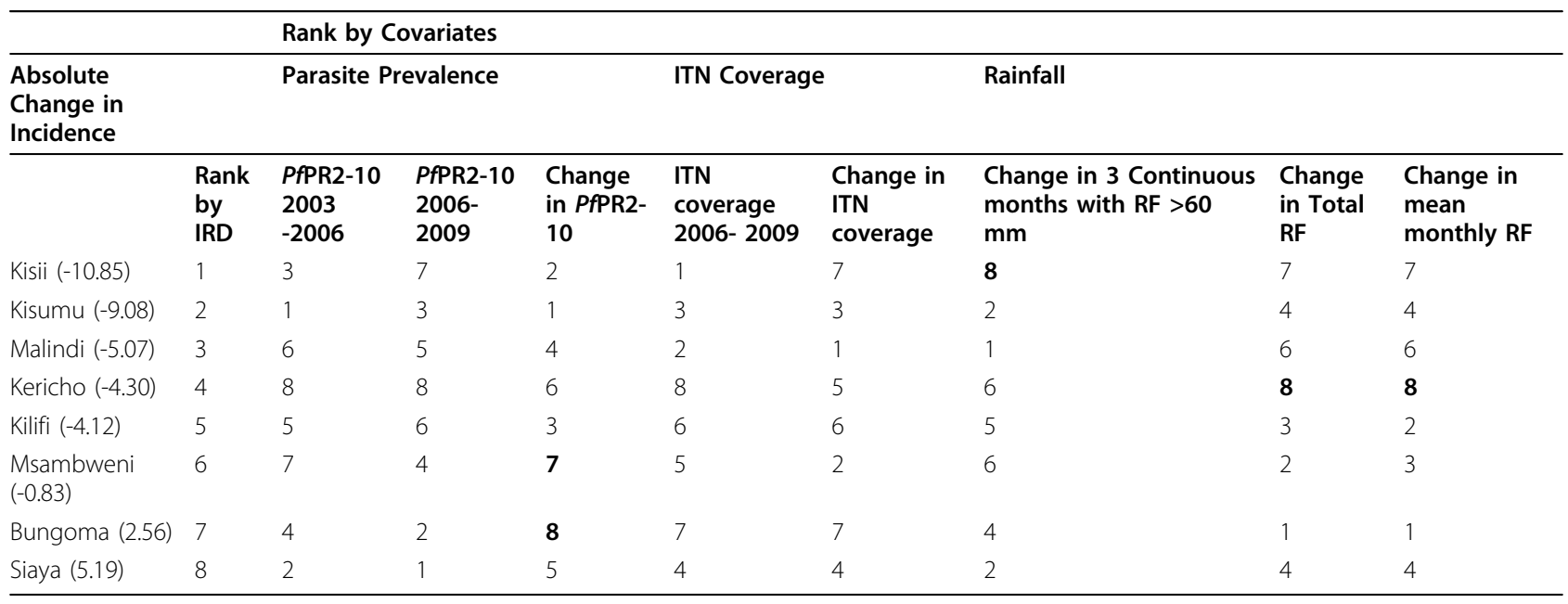

Notes:

IRD-Incidence Rate Difference.

1 = highest value/change 8 = smallest value/change.

Bold numbers represents instances where the observed trend is the reverse of the common trend i.e. an increase in transmission intensity (PfPR $\mathrm{R}_{2-10}$ ) or a reduction in rainfall.

transmission intensity in the period prior to scaled intervention for some but not all of the sites. For example Bungoma and Kisumu were ranked low and high $P f \mathrm{PR}_{2-10}$ in the first observation period. Theoretically it has been suggested that scaled intervention coverage with ITN is least likely to impact on disease incidence where starting transmission intensity is highest [44]. These data do not rule out this possibility with largest differences observed in admission rates among areas where starting $P f \mathrm{PR}_{2-10}$ was low but this trend was not consistent across the eight sites (Table 3; Figure 2). It was not possible to re-assemble spatially congruent data on drug sensitivity and drug access as a driver of temporal changes in disease incidence; nevertheless the data do show a marginal increase in the use of public health services between the two observation periods but must be balanced with the periennial problems of poor drug availability in this sector. It seems unlikely that changing to an ACT policy in September 2006 would have been a major factor in the changing incidence of malaria admissions. However, it was possible to re-construct site-specific data on ITN coverage and the changes in population coverage with an ITN were dramatic between the two observation periods (Tables 3 and 4). The absolute decline in malaria admission rates was highest in the three areas where post-scaled ITN coverage was highest (Table 3: Kisii, Kisumu and Malindi) and two of these sites also showed the highest declines in PfPR $2-10$ (Table 3: Kisii and Kisumu). Two areas where ITN coverage remained poor post-September 2006 were Bungoma and Siaya where malaria admissions increased. These observations at these sites are largely consistent with expectations [45], but there were also anomalies to this observed pattern. These anomalies do not imply that increased ITN coverage has not impacted on disease incidence rather that the explanations for temporal changes in admissions are complex and unlikely to be a result of ITN alone.

\section{Conclusions}

Support for the complex nature of change derives from the observation that most sites where declines between observation periods were observed showed evidence of changing admission rates ahead of the free mass ITN distribution campaign and prior to the implementation of ACT across Kenya. What then are the drivers of this epidemiological transition? In this paper an attempt was made to retrospectively assemble as much information as possible to develop a plausibility framework around an interrupted time-series data analysis. The conclusion is that although there have been changes, this has not been consistent across sites, both starting endemicity and ITN coverage may have accelerated declines but it is not possible to confidently identify unique factors associated with the trend in malaria admissions. In part this may well stem from having to depend on retrospective, opportunistic data assemblies to explain change. This is an unfortunate position to be in and a lesson for future monitoring and evaluation of epidemiological transitions. Without more prospectively designed plausibility studies with carefully assembled disease data congruent with information on plausible explanatory 
variables for change it may never be understood what is driving the malaria transition in Africa.

\section{Additional material}

Additional file 1: Characteristics of hospital-catchment sites Changes in Plausibility Drivers. The data provided represent the analysis of temporal changes in factors that might explain changes in disease incidence in each hospital setting and compared between hospital settings.

\section{Acknowledgements}

We are grateful to the all the district general hospital staff, who helped identify primary information and allowed access to these data. Dr James Nokes and the KEMRI team at Kilifi district hospital are thanked for the provision of the surveillance data from the hospital funded by the Wellcome Trust (UK) (\#077092). We also wish to acknowledge Sam Wareru of the Kenya Meteorological Department who provided access to monthly rainfall data, Betsy Makena who digitized the census bureau enumeration areas and Julliete Mutheu who helped assemble the hospital data. We also thank Kevin Marsh and Simon Hay for comments on earlier versions of this manuscript. This paper is published with the permission of the director KEMRI.

\section{Funding sources}

EAO and AMN are supported by the Wellcome Trust under their Research Training Fellowship programme (\#086166 and \#081829 respectively). RWS is supported by the Wellcome Trust as Principal Research Fellow (\#079080) that also supports VAA. All authors acknowledge support from the Kenya Medical Research Institute. The funders had no role in study design, data collection and analysis, decision to publish, or preparation of the manuscript.

\section{Author details}

${ }^{1}$ Malaria Public Health \& Epidemiology Group, Centre for Geographic Medicine Research - Coast, Kenya Medical Research Institute/Wellcome Trust Research Programme, P.O. Box 43640, 00100 GPO, Nairobi, Kenya. ${ }^{2}$ Centre for Tropical Medicine, Nuffield Department of Clinical Medicine, University of Oxford, CCVTM, Oxford OX3 7L, UK.

\section{Authors' contributions}

EAO was responsible for study design, data cleaning, analysis, interpretation and production of the final manuscript. VAA assisted in the primary assembly of the covariate data at each of the 8 hospitals. AMN provided temporal estimations of PfPR $2-10$ in each of the catchment areas and contributed to the final manuscript. RWS was responsible for overall scientific management, analysis, interpretation and preparation of the final manuscript. All authors read and approved the final manuscript.

\section{Competing interests}

The authors declare that they have no competing interests.

Received: 20 July 2010 Accepted: 15 October 2010

Published: 15 October 2010

\section{References}

1. Barnes Kl, Durrheim DN, Little F, Jackson A, Mehta U, Allen E, Dlamini SS, Tsoka J, Bredenkamp B, Mthembu DJ, White NJ, Sharp BL: Effect of artemether-lumefantrine policy and improved vector control on malaria burden in KwaZulu-Natal, South Africa. PLoS Med 2005, 2:e330.

2. Bhattarai A, Ali AS, Kachur SP, Martensson A, Abbas AK, Khatib R, AlMafazy AW, Ramsan M, Rotllant G, Gerstenmaier JF, Molteni F, Abdulla S, Montgomery SM, Kaneko A, Bjorkman A: Impact of artemisinin-based combination therapy and insecticide-treated nets on malaria burden in Zanzibar. PLoS Med 2007, 4:e309.

3. Kleinschmidt I, Sharp B, Benavente LE, Schwabe C, Torrez M, Kuklinski J, Morris N, Raman J, Carter J: Reduction in infection with Plasmodium falciparum one year after the introduction of malaria control interventions on Bioko Island, Equatorial Guinea. Am J Trop Med Hyg 2006, 74:972-978.

4. Nyarango PM, Gebremeskel T, Mebrahtu G, Mufunda J, Abdulmumini U, Ogbamariam A, Kosia A, Gebremichael A, Gunawardena D, Ghebrat Y, Okbaldet $Y$ : A steep decline of malaria morbidity and mortality trends in Eritrea between 2000 and 2004: the effect of combination of control methods. Malar J 2006, 5:33.

5. Okech BA, Mwobobia IK, Kamau A, Muiruri S, Mutiso N, Nyambura f, Mwatele C, Amano T, Mwandawiro CS: Use of integrated malaria management reduces malaria in Kenya. PLOS ONE 2008, 3:e4050

6. Otten M, Aregawi M, Were W, Karema C, Medin A, Jima D, Kebede W, Gausi K, Komatsu R, Korenromp E, Low-Beer D, Grabowsky M: Initial evidence of reduction of malaria cases and deaths in Rwanda and Ethiopia due to rapid scale-up of malaria prevention and treatment. Malar J 2009, 8:14.

7. Sharp BL, Kleinschmidt I, Streat E, Maharaj R, Barnes Kl, Durrheim DN, Ridl FC, Morris N, Seocharan I, Kunene S, LAG JJ, Mthembu JD, Maartens F, Martin $\mathrm{CL}$, Barreto A: Seven years of regional malaria control collaboration-Mozambique, South Africa, and Swaziland. Am J Trop Med Hyg 2007, 76:42-47.

8. Ceesay SJ, Casals-Pascual C, Erskine J, Anya SE, Duah NO, Fulford AJ, Sesay SS, Abubakar I, Dunyo S, Sey O, Palmer A, Fofana M, Corrah T, Bojang KA, Whittle HC, Greenwood BM, Conway DJ: Changes in malaria indices between 1999 and 2007 in The Gambia: a retrospective analysis. Lancet 2008, 372:1545-1554.

9. Graves PM, Osgood DE, Thomson MC, Sereke K, Araia A, Zerom M, Ceccato P, Bell M, Del Corral J, Ghebreselassie S, Brantly EP, Ghebremeskel T: Effectiveness of malaria control during changing climate conditions in Eritrea, 1998-2003. Trop Med Int Health 2008 13:218-228.

10. Teklehaimanot HD, Teklehaimanot A, Kiszewski A, Rampao HS, Sachs JD: Malaria in Sao Tome and principe: on the brink of elimination after three years of effective antimalarial measures. Am J Trop Med Hyg 2009, 80:133-140.

11. WHO: Impact of long-lasting insecticidal-treated nets (LLINs) and artemisinin-based combination therapies (ACTs) measured using surveillance data, in four African countries. Geneva: World Health Organization Global Malaria Programme 2008.

12. Okiro EA, Hay SI, Gikandi PW, Sharif SK, Noor AM, Peshu N, Marsh K, Snow RW: The decline in paediatric malaria admissions on the coast of Kenya. Malar J 2007, 6:151.

13. O'Meara WP, Bejon P, Mwangi TW, Okiro EA, Peshu N, Snow RW, Newton CR, Marsh K: Effect of a fall in malaria transmission on morbidity and mortality in Kilifi, Kenya. Lancet 2008, 372:1555-1562.

14. O'Meara WP, Mwangi TW, Williams TN, McKenzie FE, Snow RW, Marsh K. Relationship between exposure, clinical malaria, and age in an area of changing transmission intensity. Am J Trop Med Hyg 2008, 79:185-191.

15. Sievers AC, Lewey J, Musafiri P, Franke MF, Bucyibaruta BJ, Stulac SN, Rich ML, Karema C, Daily JP: Reduced paediatric hospitalizations for malaria and febrile illness patterns following implementation of community-based malaria control programme in rural Rwanda. Malar $J$ 2008, 7:167.

16. Adazu K, Hamel M, Feikin D, Ofware P, Obor D, Ogwang S, Orimba V, Vulule J, Slutsker L: Marked decline in childhood mortality in the Western Kenya DSS: Evidence from Longitudinal Data, 2003-2007. American Society of Tropical Medicine \& Hygiene, 57th Annual Meeting New Orleans, Louisiana USA 2008.

17. Okiro EA, Alegana VA, Noor AM, Mutheu JJ, Juma E, Snow RW: Malaria paediatric hospitalization between 1999 and 2008 across Kenya. BMC Med 2009, 7:75.

18. Habicht JP, Victora CG, Vaughan JP: Evaluation designs for adequacy, plausibility and probability of public health programme performance and impact. Int J Epidemiol 1999, 28:10-18.

19. Victora CG, Habicht JP, Bryce J: Evidence-based public health: moving beyond randomized trials. Am J Public Health 2004, 94:400-405.

20. Rowe AK, Steketee RW, Arnold F, Wardlaw T, Basu S, Bakyaita N, Lama M, Winston CA, Lynch M, Cibulskis RE, Shibuya K, Ratcliffe AA, Nahlen BL: Viewpoint: evaluating the impact of malaria control efforts on mortality in sub-Saharan Africa. Trop Med Int Health 2007, 12:1524-1539.

21. Box GEP, Jenkins GM, Reinsel GC: Time Series Analysis: Forecasting and Control. Upper Saddle River, NJ: Prentice-Hall, 31994. 
22. Box GEP, Tiao GC: Intervention analysis with applications to economic and environmental problems. J Am Stat Assoc 1975, 70:70-74.

23. Central Bureau of Statistics: In 1999 population and housing census: counting our people for development: Socio-economic profile of the people. Edited by: Central Bureau of Statistics. Ministry of Finance 2001:2.

24. Obonyo CO, Vulule J, Akhwale WS, Grobbee DE: In-hospital morbidity and mortality due to severe malarial anemia in western Kenya. Am J Trop Med Hyg 2007, 77(6 Suppl):23-28.

25. Berkley JA, Lowe BS, Mwangi I, Williams T, Bauni E, Mwarumba S, Ngetsa C, Slack MP, Njenga S, Hart CA, Maitland K, English M, Marsh K, Scott JA: Bacteremia among children admitted to a rural hospital in Kenya. $N$ Engl J Med 2005, 352:39-47.

26. English M, Esamai F, Wasunna A, Were F, Ogutu B, Wamae A, Snow RW, Peshu N: Assessment of inpatient paediatric care in first referral level hospitals in 13 districts in Kenya. Lancet 2004, 363(9425):1948-1953.

27. Wagner AK, Soumerai SB, Zhang F, Ross-Degnan D: Segmented regression analysis of interrupted time series studies in medication use research. $J$ Clin Pharm Ther 2002, 27(4):299-309.

28. Noor AM, Gething PW, Alegana VA, Patil AP, Hay SI, Muchiri E, Juma E, Snow RW: The risks of malaria infection in Kenya in 2009. BMC Inf Dis 2009, 9:180.

29. Smith DL, Guerra CA, Snow RW, Hay SI: Standardizing estimates of the Plasmodium falciparum parasite rate. Malar J 2007, 6:131.

30. Division of Malaria Control: National Malaria Strategy 2001-2010 Ministry of Health, Republic of Kenya 2001.

31. Noor AM, Amin AA, Akhwale WS, Snow RW: Increasing coverage and decreasing inequity in insecticide-treated bed net use among rural Kenyan children. PLoS Med 2007, 4:e255.

32. Measure-DHS: Kenya demographic \& health surveys 2003. [http://www. measuredhs.com/], Accessed June 2009.

33. Population Services International: Malaria TRaC study evaluating bed net ownership and use in 2007 in Kenya [http://www.psi.org/sites/default/files/ publication_files/766-kenya_malaria_itn_mthr_smrs.pdf], Accessed: 20 February 2010..

34. Division of Malaria Control, the Kenya National Bureau of Statistics: Kenya Malaria Indictor Survey 2007 Nairobi, Kenya: Ministry of Public Health and Sanitation 2008.

35. Shretta R, Omumbo J, Rapuoda B, Snow RW: Using evidence to change antimalarial drug policy in Kenya. Trop Med Int Health 2000, 5:755-764.

36. The efficacy of antimalarial monotherapies, sulphadoxine-pyrimethamine and amodiaquine in East Africa: implications for sub-regional policy. Trop Med Int Health 2003, 8:860-867.

37. Ogutu BR, Nzila AM, Ochong E, Mithwani S, Wamola B, Olola CH, Lowe B, Kokwaro GO, Marsh K, Newton CR: The role of sequential administration of sulphadoxine/pyrimethamine following quinine in the treatment of severe falciparum malaria in children. Trop Med Int Health 2005, 10(5):484-488.

38. Amin AA, Zurovac D, Kangwana BB, Greenfield J, Otieno DN, Akhwale WS, Snow RW: The challenges of changing national malaria drug policy to artemisinin-based combinations in Kenya. Malar J 2007, 6:72.

39. Falade C, Makanga M, Premji Z, Ortmann CE, Stockmeyer M, de Palacios PI: Efficacy and safety of artemether-lumefantrine (Coartem) tablets (sixdose regimen) in African infants and children with acute, uncomplicated falciparum malaria. Trans R Soc Trop Med Hyg 2005, 99:459-467.

40. Division of Malaria Control: Transitional plan for implementation of artemisinin-based Combination Therapy (ACT) malaria treatment policy in Kenya. Division of Malaria Control (DOMC) and Management Sciences for Health (MSH) 1-34.

41. Kangwana BB, Njogu J, Wasunna B, Kedenge SV, Memusi DN, Goodman CA, Zurovac D, Snow RW: Malaria drug shortages in Kenya: a major failure to provide access to effective treatment. Am J Trop Med Hyg 2009, 80:737-738.

42. Amin AA, Marsh V, Noor AM, Ochola SA, Snow RW: The use of formal and informal curative services in the management of paediatric fevers in four districts in Kenya. Trop Med Int Health 2003, 8:1143-1152.

43. Gitonga CW, Amin AA, Ajanga A, Kangwana BB, Noor AM, Snow RW: The use of artemether-lumefantrine by febrile children following national implementation of a revised drug policy in Kenya. Trop Med Int Health 2008, 13:487-494.
44. Smith DL, Hay SI, Noor AM, Snow RW: Predicting changing malaria risk after expanded insecticide-treated net coverage in Africa. Trends Parasitol 2009, 25:511-516.

45. Nevill CG, Some ES, Mung'ala VO, Mutemi W, New L, Marsh K, Lengeler C, Snow RW: Insecticide-treated bednets reduce mortality and severe morbidity from malaria among children on the Kenyan coast. Trop Med Int Health 1996, 1:139-146.

46. East African Network for Monitoring Antimalarial Treatment EANMAT: Monitoring antimalarial drug resistance within national malaria control programmes: the EANMAT experience. Trop Med Int Health 2001, 6:891-898.

47. Obonyo CO, Ochieng F, Taylor WR, Ochola SA, Mugitu K, Olliaro P, ter Kuile F, Oloo AJ: Artesunate plus sulfadoxine-pyrimethamine for uncomplicated malaria in Kenyan children: a randomized, double-blind, placebo-controlled trial. Trans R Soc Trop Med Hyg 2003, 97:585-591.

48. Abdulla S, Sagara I, Borrmann S, D'Alessandro U, Gonzalez R, Hamel M, Ogutu B, Martensson A, Lyimo J, Maiga H, Sasi P, Nahum A, Bassat Q, Juma E, Otieno L, Bjorkman A, Beck HP, Andriano K, Cousin M, Lefevre G, Ubben D, Premji Z: Efficacy and safety of artemether-lumefantrine dispersible tablets compared with crushed commercial tablets in African infants and children with uncomplicated malaria: a randomised, singleblind, multicentre trial. Lancet 2008, 372:1819-1827.

49. Bassat $Q$, Mulenga M, Tinto H, Piola P, Borrmann S, Menendez C, Nambozi M, Valea I, Nabasumba C, Sasi P, Bacchieri A, Corsi M, Ubben D, Talisuna A, D'Alessandro U: Dihydroartemisinin-piperaquine and artemether-lumefantrine for treating uncomplicated malaria in African children: a randomised, non-inferiority trial. PLOS ONE 2009, 4(11):e7871.

50. Borrmann S, Sallas WM, Machevo S, Gonzalez R, Bjorkman A, Martensson A, Hamel M, Juma E, Peshu J, Ogutu B, Djimde A, D'Alessandro U, Marrast AC, Lefevre G, Kern SE: The effect of food consumption on lumefantrine bioavailability in African children receiving artemether-lumefantrine crushed or dispersible tablets (Coartem) for acute uncomplicated Plasmodium falciparum malaria. Trop Med Int Health 15:434-441.

51. Juma EA, Obonyo CO, Akhwale WS, Ogutu BR: A randomized, open-label, comparative efficacy trial of artemether-lumefantrine suspension versus artemether-lumefantrine tablets for treatment of uncomplicated Plasmodium falciparum malaria in children in western Kenya. Malar J 2008, 7:262.

52. Mens PF, Sawa P, van Amsterdam SM, Versteeg I, Omar SA, Schallig HD, Kager PA: A randomized trial to monitor the efficacy and effectiveness by QT-NASBA of artemether-lumefantrine versus dihydroartemisininpiperaquine for treatment and transmission control of uncomplicated Plasmodium falciparum malaria in western Kenya. Malar J 2008, 7:237.

53. Premji Z, Umeh RE, Owusu-Agyei S, Esamai F, Ezedinachi EU, Oguche S, Borrmann S, Sowunmi A, Duparc S, Kirby PL, Pamba A, Kellam L, Guiguemde R, Greenwood B, Ward SA, Winstanley PA: Chlorproguanildapsone-artesunate versus artemether-lumefantrine: a randomized, double-blind phase III trial in African children and adolescents with uncomplicated Plasmodium falciparum malaria. PLOS ONE 2009, 4:e6682.

doi:10.1186/1475-2875-9-285

Cite this article as: Okiro et al.: Changing malaria intervention coverage, transmission and hospitalization in Kenya. Malaria Journal 2010 9:285.

\section{Submit your next manuscript to BioMed Central and take full advantage of:}

- Convenient online submission

- Thorough peer review

- No space constraints or color figure charges

- Immediate publication on acceptance

- Inclusion in PubMed, CAS, Scopus and Google Scholar

- Research which is freely available for redistribution

Submit your manuscript at www.biomedcentral.com/submit
C Biomed Central 